\title{
Sinonasal sarcoidosis: a case report
}

\author{
Selma Erdoğan Düzcüํㅜ, Serap Köybaşı Şanal \\ ${ }^{1}$ Department of Pathology, Bolu Abant İzzet Baysal University School of Medicine, İzzet Baysal Training and Research Hospital, Bolu, \\ Turkey \\ ${ }^{2}$ Department of Otorhinolaryngology, Bolu Abant İzet Baysal University School of Medicine, İzet Baysal Training and Research \\ Hospital, Bolu, Turkey
}

\section{ABSTRACT}

Objectives: Sarcoidosis is an idiopathic and multisystemic chronic disease characterized by non-caseating granulomas. Sinonasal sarcoidosis may be seen in an isolated form or related to a multisystemic disease. A 48year-old female patient visited the otolaryngology department with complaints of headache and nasal obstruction for two years. The physical examination revealed a saddling on the nose, and crusted fragile mucosa was observed bilaterally in nasal endoscopy. Paranasal CT showed invasion of the right orbita. In chest X-ray, there was bilateral hilar enlargement, and lung biopsy revealed non-caseating granulomas. A bilateral endoscopic sinus surgery was applied to the patient. Upon histopathological demonstration of multiple noncaseating granulomas in the specimen, the diagnosis of sinonasal sarcoidosis was made. The patient responded well to the following steroid treatment. Although rare, sarcoidosis should be taken into consideration in differential diagnosis of sinonasal diseases.

Keywords: sarcoidosis, sinonasal involvement, non-caseating granulomas

$\mathrm{S}^{\mathrm{a}}$ arcoidosis is an idiopathic and multisystemic chronic disease characterized by non-caseating granulomas $[1,2]$. The primary site of involvement is the lung [3]. It mostly affects young and middle-aged women [4].

The pathological background of sarcoidosis is not clear. It is thought to be triggered by an oligoclonal CD4 positive $\mathrm{T}$ cell response to an unknown antigen with the effects of environmental factors (e.g. bacteria, viruses, fungi, bugs, inorganic particles) $[5,6]$.

Sinonasal sarcoidosis may be seen in an isolated form or related to a multisystemic disease [2]. Nasal localization constitutes less than $2 \%$ of cases and is rare [7]. A case of nasal sarcoidosis alongside systemic attachment was first reported by Boeck in 1905 [2]. Its otorhinolaryngologic signs and symptoms are not specific and are similar to the signs of other more common diseases [9]. The most common symptom is nasal obstruction [4]. The diagnosis of sarcoidosis is made through clinical, radiologic and histologic examinations together [7].

The aim of this case presentation is to report the clinicopathologic characteristics of a sinonasal sarcoidosis case as a part of a multisystemic disease.

\section{CASE PRESENTATION}

A 48-year-old female patient admitted the otolaryngology department with complaints of nasal obstruction and headache for two years. The patient's medical history revealed neither allergies nor usage of 


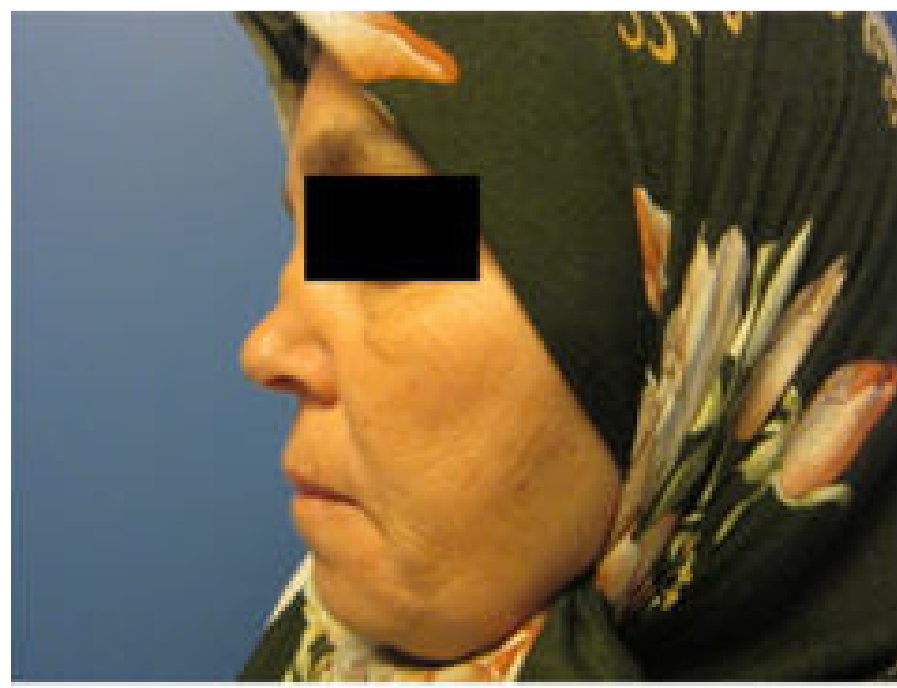

Fig. 1. Saddle nose appearance.

alcohol or tobacco.

The external nose had a saddle nose appearance (Fig. 1). In the endoscopic examination of the nose, the bilateral nose, septum, nasal lateral wall, inferior concha mucosa had crusted granular and fragile appearance. The nostrils were full of granular tissue. The right middle concha was eroded (Fig. 2).

Paranasal CT revealed soft tissue density in both anterior and posterior ethmoidal cells and the maxillary sinuses. The lamina papyracea was observed to be eroded, and the medial rectus muscle was compressed. A bilateral functional endoscopic sinus surgery (FESS) was planned. In the preoperative work

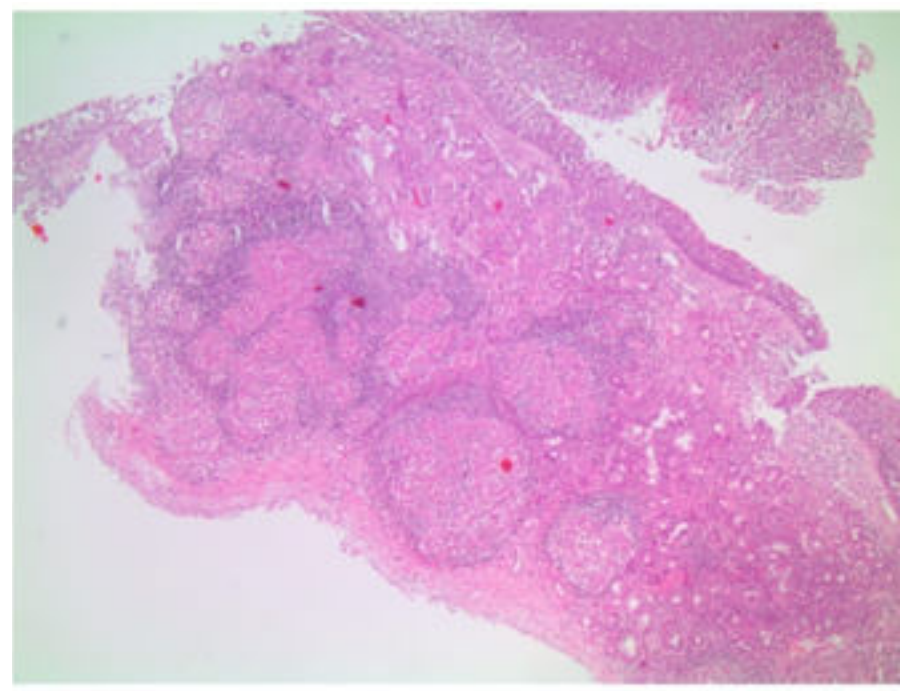

Fig. 3. The view of granulomas under the respiratory epithelium (H-E staining, $\times 40)$.

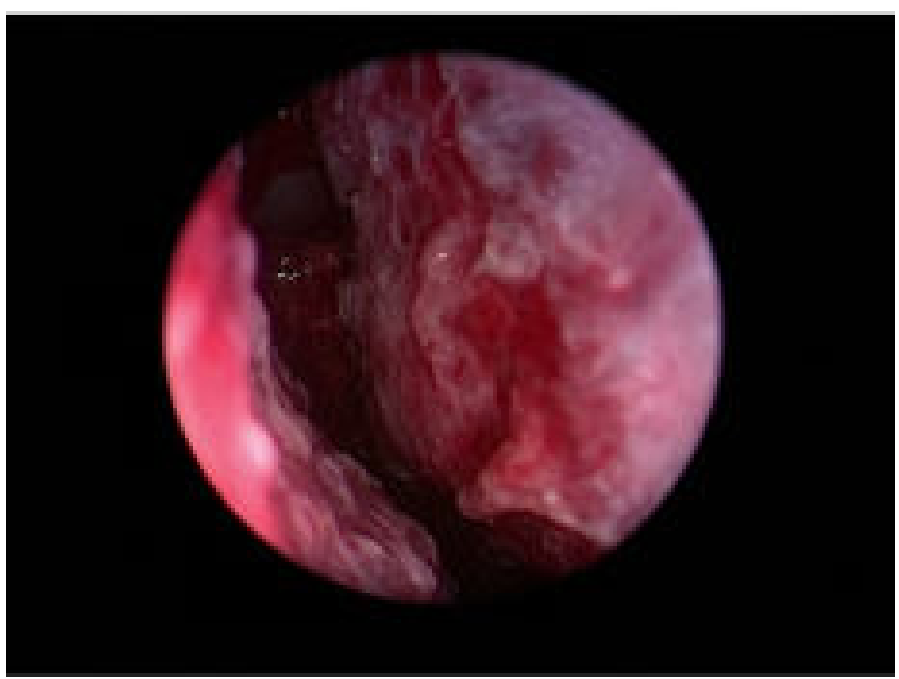

Fig. 2. In the endoscopic examination of the nose, the nostrils were full of granular tissue.

up, a pulmonology consultation was performed because of bilateral hilar enlargement. Thorax CT showed bilateral hilar, mediastinal lymphadenopathy and nodular opacity. Non-caseating granulomas were diagnosed via lungbiopsy that was taken endobronchially at an external medical center.

A bilateral functional endoscopic sinus surgery was applied both to get a histopathological diagnosis and avoid any possible orbital complication of the disease such as diplopia. The granulomatous tissue that filled the nasal cavity and obliterated the bilateral posteroanterior ethmoidal cells and the maxillary sinuses was removed. The right lamina papyracea was

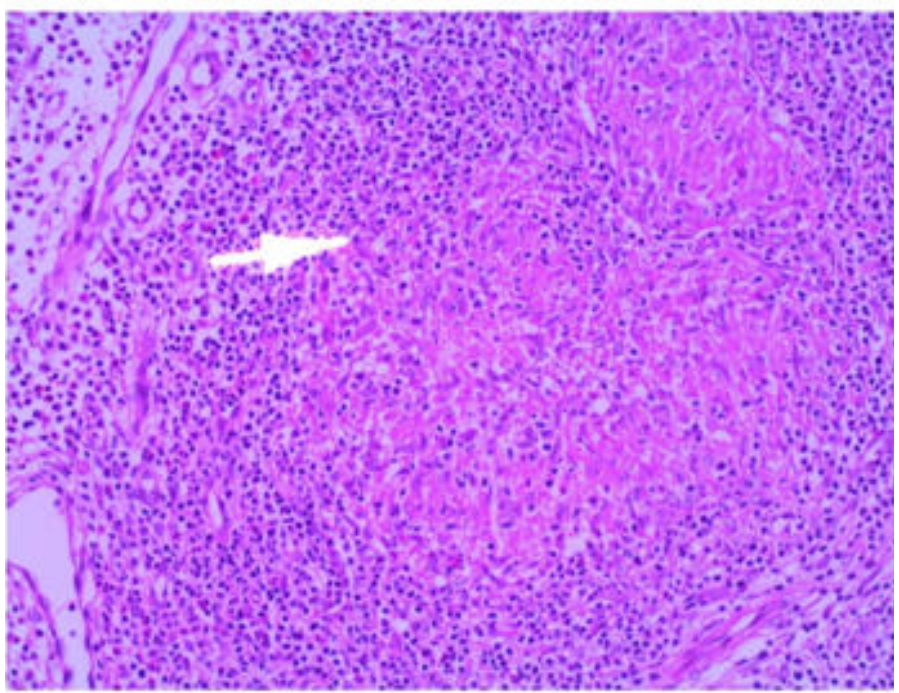

Fig. 4. The granuloma was composed of epitheloid histiocytes, mature mononuclear cells (lymphocytes)(H-E staining, $\times 200)$. 


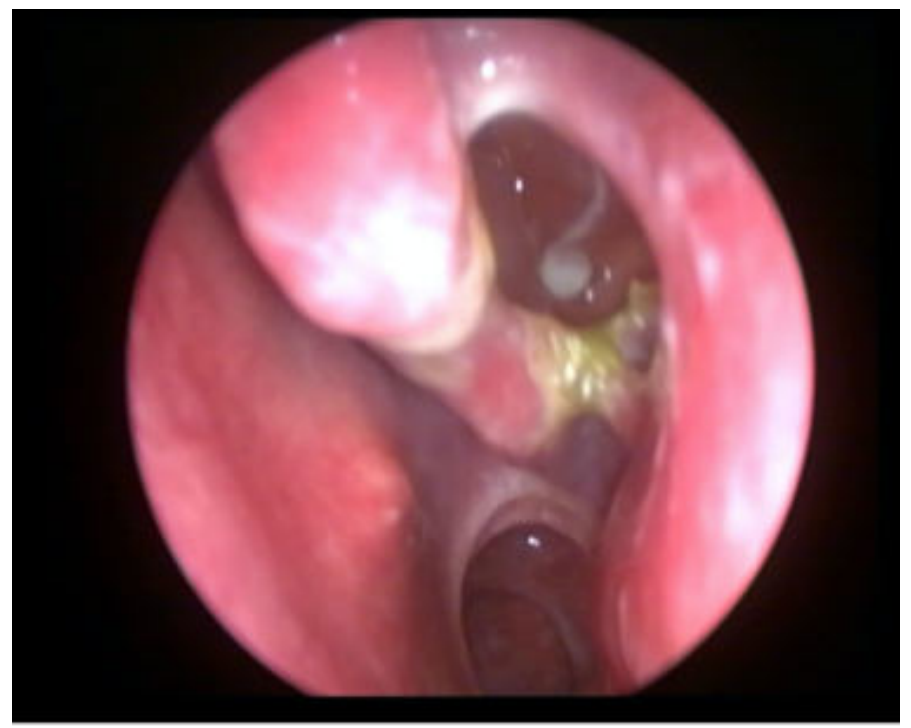

Fig. 2. In the endoscopic examination of the nose, the nostrils were full of granular tissue.

observed to be eroded, and the pressure over the right orbita was released. Repairing the saddle nose deformity was postponed to another operation after the histological diagnosis and medical treatment.

In the microscopic examination performed in the pathology department, mature mononuclear cells (lymphocytes), epithelioid histiocytes and Langhanstype multinuclear giant cell multiple granuloma structures were observed. No caseification necrosis or suppurative necrosis was seen in the granulomas [Figs. 3 and 4]. Since the observation of a non-caseating granulomatous inflammation suggested sarcoidosis, a differential diagnosis was required. Although the absence of caseification made the diagnosis of tuberculosis less likely, an additional EZN (EhrlichZiehl-Neelsen) staining was applied, and no mycobacteria were observed. No staining was observed with the PAS histochemical stain, which is applied for fungi, either. Combining these histopathological findings with the clinical presentation, a diagnosis of sarcoidosis was made. The patient received steroid treatment starting with $40 \mathrm{mg}$ per day and tapering the dose in weeks. The bilateral hilar enlargement was observed to respond well.

In the ENT evaluation, the complaints of the patient were completely relieved. In the endoscopic nasal examination, granular whitish mucosa was seen to turn into a normal appearance in color and nature, with tiny polypoid structures observed in the ethmoidectomy cavities (Fig. 5).

\section{DISCUSSION}

Sarcoidosis is a complex disorder in which head and neck involvement may be observed in $10-15 \%$ of the cases; however, sinonasal involvement is reported in about only $1 \%$ of cases of sarcoidosis [10].

Sarcoidosis may involve both the mucosa and cartilaginous and bony structures beneath the mucosa in the nose and the paranasal sinuses. Despite the presence of clinical findings, imaging and tissue diagnosis are needed for confirmation of the disease. It is important to understand and differentiate sarcoidosis as it may involve vital structures in this area [11].

Sinonasal sarcoidosis most often attaches to the septum mucosa and the inferior turbinate. In some patients, nasal bone lesions may also be seen. Sinonasal sarcoidosis may be clinically classified as atrophic, hypertrophic, destructive and nasally widening [12]. In our case, it could be accepted to be both hypertrophic and destructive.

In some cases, as in ours, nasal involvement may be the first sign followed by pulmonary disease [4]. A tissue biopsy is necessary to ascertain the diagnosis [4]. The observation of opacification and the presence of non-caseating granuloma are important in its discrimination from other granulomatous diseases [13]. Beside non-caseating granuloma structures, hyaline fibrosis and necrosis may also be seen in the histopathology [4]. In our case, focal necrosis was also present. In sarcoid granuloma, wide, pale epithelioid histiocytes with oval nuclei are observed, and $\mathrm{T}$ lymphocytes may be seen to surround the histiocytes [14]. Additionally, multinuclear Langhans-type giant cells may be observed $[6,10]$. The giant cells seen in sarcoidosis may be larger than the giant cells seen in tuberculosis, and they may have more nuclei [6]. In the giant cells, intracytoplasmic inclusion objects known as asteroid and Schaumann bodies may be found [6].

Differential diagnosis of sinonasal sarcoidosis includes Wegener granulomatosis, fungal infections, foreign body reactions and lymphoma [14]. In the histopathology of Wegener granulomatosis, the presence of leukocytoclastic vasculitis or necrotizing granulomas help differentiate the disease [13]. In James et al.'s [8]retrospective analysis with 1686 patients, $8.3 \%$ patients were noted non-pulmonary 
sarcoidosis with biopsy proven. Among the 1944 different sinus surgery materials Van der Boer et al. [15] reported, only 3 patients were reported to have sarcoidosis. Since the suspicion of clinical sarcoidosis was supported with pathological examination, the necessity of FESS was stressed [15]. Our patient had both septal and paranasal sinus involvement. Braun et al. [2] reported 15 (8 male, 7 female)sinonasal sarcoidosis cases. In one of those cases, a saddle nose deformity was present in similarity to our case. In 12 patients, there were sarcoidosis in the sinonasal area and the lungs [2]. Kirsten et al. [1] reported 12 (4 females, 8 males) sinonasal sarcoidosis cases proven through biopsy. All of the cases were applied sinus surgery and corticosteroid treatments similar to our case. The most common attachment location in these patients was the maxillary sinus. All cases had lymph nodes and pulmonary involvement [1].

In a case of sinonasal sarcoidosis in a 48-year-old male patient reported by Mazziotti et al. [4], perineural spreading was observed around the trigeminal and Vidian nerves. In the nasal mucosa biopsy, noncaseating granulomas were seen, and through the presence of hilar lymphadenopathy, the diagnosis of sarcoidosis was supported [3].

Systemic or topical use of steroids is commonly used modalities in treatment of sinonasal sarcoidosis [4]. However endoscopic sinus surgery may also be applied in selected cases such as intraorbital complication risk, massive disease and in case of medical treatment failure [4]. In our case, we applied surgery because of the massive granulations and intraorbital invasion risk. Additional topical steroids were applied to help control the local disease and systemic steroids for the pulmonary component [4]. Sinus surgery may decrease the need for systemic steroid use [4]. Gulati et al. [11] reported seven cases of sinonasal sarcoidosis, three of whom were treated with only systemic prednisolone. The remaining four cases were reported to undergo sinus surgery and topical steroids treatments [11]. In cases where the use of systemic corticosteroids is contraindicated, methotrexate is also stated to be usable [14].

\section{CONCLUSION}

Sinonasal involvement of sarcoidosis is a rare condition. Nasal crusting and obstruction being the most common complaints, this may be confused with other inflammatory nasal diseases, so differential diagnosis is important. It is usually accompanied by pulmonary involvement; however, it may be seen as isolated in that area. Treatment of sinonasal sarcoidosis depends upon the site and extent of the disease including oral and/or intranasal steroids, and in some cases, low-dose methotrexate. Sinus surgery may be necessary in the management of selected cases.

\section{Informed consent}

Written informed consent was obtained from the patient for publication of this case report and any accompanying images.

\section{Conflict of interest}

The authors declared that there are no potential conflicts of interest with respect to the research, authorship, and/or publication of this article.

\section{Authors' Contributions}

Concept - S.E.D., S.K.Ş.; Design - S.E.D., S.K.; Supervision - S.E.D., S.K.; Resource - S.E.D., S.K.; Materials - S.E.D., S.K.; Data Collection and/ or Processing - S.E.D., S.K.; Analysis and/or Interpretation - S.E.D., S.K.; Literature Search S.E.D., S.K.; Writing - S.E.D., S.K.; Critical Reviews - S.E.D., S.K.

\section{REFERENCES}

1. Kirsten AM, Watz H, Kirsten D. Sarcoidosis with involvement of the paranasal sinuses - a retrospective analysis of 12 biopsyproven cases. BMC Pulm Med 2013;13:59.

2. Braun JJ, Gentine A and Pauli G. Sinonasal sarcoidosis: review and report of fifteen cases. Laryngoscope 2004;114:1960-3.

3. Mazziotti S, Gaeta M, Blandino A, Vinci S, Pandolfo I, Perineural spread in a case of sinonasal sarcoidosis: case report. AJNR Am J Neuroradiol, 2001;22:1207-8.

4. Erbek S, Erbek SS, Tosun E, Çakmak O. A rare case sarcoidosis involving the middle turbinates: an incidental diagnosis. Diagn Pathol 2006;1:44.

5. Bianchini C, Tosin E, Ciorba A, Pelucchi S, Pastore A. Subcutaneoussarcoidosis: a rare involvement of the paralateral nasal region. Acta Otorhinolaryngol Ital 2011;31:118-20.

6. Mekhail P, Abo-Khatwa M, El-Hawary A, Khan A. Cutaneous nasal nodule: a warning sign for sinonasal sarcoidosis. Internet J Head Neck Surg 2008;3(1). 
7. Mahfoudhi M, Khammassi K, Turki S, Salah MB. Systemic sarcoidosis revealed by a nasal lesion: a case report. Int J Clin Med 2015;6:257.

8. James WE, Koutroumpakis E, Saha B, Nathani A, Saavedra $\mathrm{L}$, Yucel RM, et al. Clinical features of extrapulmonary sarcoidosis without lung involvement. Chest 2018;154:349-56.

9. Dessouky OY. Isolated sinonasal sarcoidosis with intracranial extension: case report. Acta Otorhinolaryngol Ital 2008;2:306-8. 10. McCaffrey TV, McDonald TJ. Sarcoidosis of the nose and paranasal sinuses. Laryngoscope 1983;93:1281-4.

11. Gulati S, Krossnes B, Olofsson J, Danielsen A. Sinonasal involvement in sarcoidosis: a report of seven cases and review of literature. Eur Arch Otorhinolaryngol 2012;269:891-6.

12. Badhey AK, Kadakia S, Carrau RL, Iacob C, Khorsandi A. Sarcoidosis of the head and neck. Head Neck Pathol 2015;9:2608.

13. Helliwell TR. Non-infectious inflammatory lesions of the sinonasal tract. Head Neck Pathol 2016;10:32-9.

14. McCaffrey TV. Nasal manifestations of systemic dieseases. Otolaryngol Pol 2009;63:228-35.

15. Van den Boer $C$, Brutel G, de Vries N. Is routine histopathological examination of FESS material useful? Eur Arch Otorhinolaryngol 2010;267:381-4. 